\title{
ERROR ESTIMATES FOR THE NUMERICAL APPROXIMATION OF BOUNDARY SEMILINEAR ELLIPTIC CONTROL PROBLEMS. CONTINUOUS PIECEWISE LINEAR APPROXIMATIONS
}

\author{
E. Casas, ${ }^{1}$ and M. Mateos ${ }^{2}$ \\ ${ }^{1}$ Dpto. de Matemática Aplicada y Ciencias de la Computación, E.T.S.I. Industriales y de Tele- \\ comunicación, Universidad de Cantabria, 39071 Santander, Spain, eduardo.casas@unican.es*, \\ ${ }^{2}$ Dpto. de Matemáticas, E.P.S.I. de Gijón, Universidad de Oviedo, Campus de Viesques, 33203 \\ Gijón,Spain,mmateos@uniovies
}

\begin{abstract}
We discuss error estimates for the numerical analysis of Neumann boundary control problems. We present some known results about piecewise constant approximations of the control and introduce some new results about continuous piecewise linear approximations. We obtain the rates of convergence in $L^{2}(\Gamma)$. Error estimates in the uniform norm are also obtained. We also discuss the semidiscretization approach as well as the improvement of the error estimates by making an extra assumption over the set of points corresponding to the active control constraints.
\end{abstract}

keywords: Boundary control, semilinear elliptic equation, numerical approximation, error estimates.

\section{Introduction}

This paper continues a series of works about error estimates for the numerical analysis of control problems governed by semilinear elliptic partial differential equations. In [1] a distributed problem approximated by piecewise constant controls was studied. In [7] the control appears in the boundary. This makes the task more difficult since the states are now less regular than in the distributed case. Piecewise constant approximations were used in that reference. The advantage of these is that we have a pointwise expression both for the control and its approximation, which we can compare to get uniform convergence. The reader is addressed to these papers for further references about error estimates for the approximation of linear-quadratic problems governed by partial differ-

*Paper written with financial support of Ministerio de Ciencia y Tecnología (Spain).

Please use the following format when citing this chapter:

Casas, E., and Mateos, M., 2006, in IFIP International Federation for Information Processing, Volume 202, Systems, Control, Modeling and Optimization, eds.

Ceragioli, F., Dontchev, A., Furuta, H., Marti, K., Pandolfi, L., (Boston: Springer), pp. 91-101. 
ential equations and for the approximation of control problems governed by ordinary differential equations.

In the case of continuous piecewise linear approximations of the control, there exists not such a pointwise formula in general. If the functional is quadratic with respecto to the control, recent results in [8] about the stability of $L^{2}$ projections in Sobolev $W^{s, p}(\Gamma)$ spaces allow us to obtain uniform convergence and adapt the proofs. The general case is more delicate. Results for distributed control problems can be found in [3]. The main purpose of this paper is to obtain similar results for Neumann boundary controls. This is done in Theorem 10 .

We also refer to the works for distributed linear-quadratic problems about semidiscretization [9] and postprocessing [10]. The first proposes only discretizing the state, and not the control. The solution can nevertheless be expressed with a finite number of parameters via the adjoint-state and the problem can be solved with a computer with a slightly changed optimization code. The second one proposes solving a completely discretized problem with piecewise constant approximations of the control and finally construct a new control using the pointwise projection of the discrete adjoint state. We are able to reproduce the first scheme for Neumann boundary controls, a general functional and a semilinear equation.

The rest of the paper is as follows. In the next section, we define precisely the problem. In Section 3 we recall several results about this control problem. Section 4 contains the main results of this paper: we discretize the problem and obtain error estimates for the solutions.

\section{Statement of the problem}

Throughout the sequel, $\Omega$ denotes an open convex bounded polygonal set of $\mathbf{R}^{2}$ and $\Gamma$ is the boundary of $\Omega$. We will also take $p>2$. In this domain we formulate the following control problem

$$
(\mathrm{P})\left\{\begin{array}{l}
\inf J(u)=\int_{\Omega} L\left(x, y_{u}(x)\right) d x+\int_{\Gamma} l\left(x, y_{u}(x), u(x)\right) d \sigma(x) \\
\text { subject to }\left(y_{u}, u\right) \in H^{1}(\Omega) \times L^{\infty}(\Gamma) \\
u \in U^{a d}=\left\{u \in L^{\infty}(\Gamma) \mid \alpha \leq u(x) \leq \beta \text { a.e. } x \in \Gamma\right\} \\
\left(y_{u}, u\right) \text { satisfying the state equation }(1)
\end{array}\right.
$$

$$
\left\{\begin{aligned}
-\Delta y_{u}(x) & =a_{0}\left(x, y_{u}(x)\right) & & \text { in } \Omega \\
\partial_{\nu} y_{u}(x) & =b_{0}\left(x, y_{u}(x)\right)+u(x) & & \text { on } \Gamma,
\end{aligned}\right.
$$

where $-\infty<\alpha<\beta<+\infty$. Here $u$ is the control while $y_{u}$ is said to be the associated state. The following hypotheses are assumed about the functions involved in the control problem $(\mathrm{P})$ :

(A1) The function $L: \Omega \times \mathbf{R} \longrightarrow \mathbf{R}$ is measurable with respect to the first component, of class $C^{2}$ with respect to the second, $L(\cdot, 0) \in L^{1}(\Omega)$, 
$\frac{\partial L}{\partial y}(\cdot, 0) \in L^{p}(\Omega) \frac{\partial^{2} L}{\partial y^{2}}(\cdot, 0) \in L^{\infty}(\Omega)$ and for all $M>0$ there exists a constant $C_{L, M}>0$ such that $\left|\frac{\partial^{2} L}{\partial y^{2}}\left(x, y_{2}\right)-\frac{\partial^{2} L}{\partial y^{2}}\left(x, y_{1}\right)\right| \leq C_{L, M}\left|y_{2}-y_{1}\right|$, for a.e. $x \in \Omega$ and $|y|,\left|y_{i}\right| \leq M, i=1,2$.

(A2) The function $l: \Gamma \times \mathbf{R}^{2} \longrightarrow \mathbf{R}$ is Lipschitz with respect to the first component, of class $C^{2}$ with respect to the second and third variables, $l(\cdot, 0,0) \in$ $L^{1}(\Gamma), D_{(y, u)}^{2} l(\cdot, 0,0) \in L^{\infty}(\Gamma)$ and for all $M>0$ there exists a constant $C_{l, M}>0$ such that

$$
\begin{gathered}
\left|\frac{\partial l}{\partial y}\left(x_{2}, y, u\right)-\frac{\partial l}{\partial y}\left(x_{1}, y, u\right)\right|+\left|\frac{\partial l}{\partial u}\left(x_{2}, y, u\right)-\frac{\partial l}{\partial u}\left(x_{1}, y, u\right)\right| \leq C_{l, M}\left|x_{2}-x_{1}\right|, \\
\left\|D_{(y, u)}^{2} l\left(x, y_{2}, u_{2}\right)-D_{(y, u)}^{2} l\left(x, y_{1}, u_{1}\right)\right\| \leq C_{l, M}\left(\left|y_{2}-y_{1}\right|+\left|u_{2}-u_{1}\right|\right),
\end{gathered}
$$

for a.e. $x, x_{i} \in \Gamma$ and $|y|,\left|y_{i}\right|,|u|,\left|u_{i}\right| \leq M, i=1,2$, where $D_{(y, u)}^{2} l$ denotes the second derivative of $l$ with respect to $(y, u)$. Moreover we assume that there exists $\Lambda>0$ such that

$$
\frac{\partial^{2} l}{\partial u^{2}}(x, y, u) \geq \Lambda, \text { a.e. } x \in \Gamma \text { and }(y, u) \in \mathbf{R}^{2} .
$$

Let us remark that this inequality implies the strict convexity of $l$ with respect to the third variable.

(A3) The function $a_{0}: \Omega \times \mathbf{R} \longrightarrow \mathbf{R}$ is measurable with respect to the first variable and of class $C^{2}$ with respect to the second, $a_{0}(\cdot, 0) \in L^{p}(\Omega)$, $\frac{\partial a_{0}}{\partial y}(\cdot, 0) \in L^{\infty}(\Omega), \frac{\partial^{2} a_{0}}{\partial y^{2}}(\cdot, 0) \in L^{\infty}(\Omega), \frac{\partial a_{0}}{\partial y}(x, y) \leq 0$ a.e. $x \in \Omega$ and $y \in$ $\mathbf{R}$ and for all $M>0$ there exists a constant $C_{a_{0}, M}>0$ such that $\left|\frac{\partial^{2} a_{0}}{\partial y^{2}}\left(x, y_{2}\right)-\frac{\partial^{2} a_{0}}{\partial y^{2}}\left(x, y_{1}\right)\right|<C_{a_{0}, M}\left|y_{2}-y_{1}\right|$ a.e. $x \in \Omega$ and $\left|y_{1}\right|,\left|y_{2}\right| \leq M$.

(A4) The function $b_{0}: \Gamma \times \mathbf{R} \longrightarrow \mathbf{R}$ is Lipschitz with respect to the first variable and of class $C^{2}$ with respect to the second, $b_{0}(\cdot, 0) \in W^{1-1 / p, p}(\Gamma)$, $\frac{\partial^{2} b_{0}}{\partial y^{2}}(\cdot, 0) \in L^{\infty}(\Gamma), \frac{\partial b_{0}}{\partial y}(x, y) \leq 0$ and for all $M>0$ there exists a constant $C_{b_{0}, M}>0$ such that

$$
\left|\frac{\partial b_{0}}{\partial y}\left(x_{2}, y\right)-\frac{\partial b_{0}}{\partial y}\left(x_{1}, y\right)\right| \leq C_{b_{0}, M}\left|x_{2}-x_{1}\right|
$$




$$
\left|\frac{\partial^{2} b_{0}}{\partial y^{2}}\left(x, y_{2}\right)-\frac{\partial^{2} b_{0}}{\partial y^{2}}\left(x, y_{1}\right)\right| \leq C_{b_{0}, M}\left|y_{2}-y_{1}\right|
$$

for a.e. $x, x_{1}, x_{2} \in \Gamma$ and $|y|,\left|y_{1}\right|,\left|y_{2}\right| \leq M$.

(A5) At least one of the two conditions must hold: either $\frac{\partial a_{0}}{\partial y}(x, y)<0$ in $E_{\Omega} \times \mathbf{R}$ with $E_{\Omega} \subset \Omega$ of positive $n$-dimensional measure or $\frac{\partial b_{0}}{\partial y}(x, y)<0$ on $E_{\Gamma} \times \mathbf{R}$ with $E_{\Gamma} \subset \Gamma$ of positive $(n-1)$-dimensional measure.

\section{Analysis of the control problem}

Let us briefly state some useful results known for this control problem. The proofs can be found in [7].

THEOREM 1 For every $u \in L^{2}(\Gamma)$ the state equation (1) has a unique solution $y_{u} \in H^{3 / 2}(\Omega)$, that depends continuously on $u$. Moreover, there exists $p_{0}>2$ depending on the measure of the angles in $\Gamma$ such that if $u \in W^{1-1 / p, p}(\Gamma)$ for some $2 \leq p \leq p_{0}$, then $y_{u} \in W^{2, p}(\Omega)$.

Let us note that the inclusion $H^{3 / 2}(\Omega) \subset C(\bar{\Omega})$ holds for Lipschitz domains in $\mathbf{R}^{2}$. As a consequence of the theorem above, we know that the functional $J$ is well defined in $L^{2}(\Gamma)$. Let us discuss the differentiability properties of $J$.

THEOREM 2 Suppose that assumptions (A3)-(A4) are satisfied. Then the mapping $G: L^{\infty}(\Gamma) \longrightarrow H^{3 / 2}(\Omega)$ defined by $G(u)=y_{u}$ is of class $C^{2}$. Under the assumptions $(A l)-(A 4)$, the functional $J: L^{\infty}(\Gamma) \rightarrow \mathbf{R}$ is of class $C^{2}$. Moreover, for every $u, v \in L^{\infty}(\Gamma)$

$$
\int_{\Gamma}\left(\frac{\partial l}{\partial u}\left(x, y_{u}, u\right)+\varphi_{u}\right) v d \sigma
$$

where the adjoint state $\varphi_{u} \in H^{3 / 2}(\Omega)$ is the unique solution of the problem

$$
\left\{\begin{array}{l}
-\Delta \varphi=\frac{\partial a_{0}}{\partial y}\left(x, y_{u}\right) \varphi+\frac{\partial L}{\partial y}\left(x, y_{u}\right) \text { in } \Omega \\
\partial_{\nu} \varphi=\frac{\partial b_{0}}{\partial y}\left(x, y_{u}\right) \varphi+\frac{\partial l}{\partial y}\left(x, y_{u}, u\right) \text { on } \Gamma .
\end{array}\right.
$$

Expressions for the derivatives of $G$ and the second derivative of $J$ can be found in [7].

The existence of a solution for problem (P) follows easily from our assumptions (A1)-(A5). In particular, we underline the important fact that the function $l$ is convex with respect to the third variable. See (2). The first order optimality conditions for Problem (P) follow readily from Theorem 2. 
THEOREM 3 Assume that $\bar{u}$ is a local solution of Problem $(P)$. Then there exist $\bar{y}, \bar{\varphi} \in H^{3 / 2}(\Omega)$ such that

$$
\begin{gathered}
\left\{\begin{aligned}
&-\Delta \bar{y}(x)=a_{0}(x, \bar{y}(x)) \text { in } \quad \Omega \\
& \partial_{\nu} \bar{y}(x)=b_{0}(x, \bar{y}(x))+\bar{u}(x) \quad \text { on } \quad \Gamma
\end{aligned}\right. \\
\left\{\begin{aligned}
-\Delta \bar{\varphi}=\frac{\partial a_{0}}{\partial y}(x, \bar{y}) \bar{\varphi}+\frac{\partial L}{\partial y}(x, \bar{y}) \quad \text { in } \quad \Omega \\
\partial_{\nu} \bar{\varphi}=\frac{\partial b_{0}}{\partial y}(x, \bar{y}) \bar{\varphi}+\frac{\partial l}{\partial y}(x, \bar{y}, \bar{u}) \quad \text { on } \quad \Gamma
\end{aligned}\right. \\
\int_{\Gamma}\left(\frac{\partial l}{\partial u}(x, \bar{y}, \bar{u})+\bar{\varphi}\right)(u-\bar{u}) d \sigma(x) \geq 0 \quad \forall u \in U^{a d}
\end{gathered}
$$

First order optimality conditions allow us to deduce extra regularity for the optimal control.

THEOREM 4 Suppose that $\bar{u}$ is a local solution of $(P)$, then for all $x \in \Gamma$ the equation

$$
\bar{\varphi}(x)+\frac{\partial l}{\partial u}(x, \bar{y}(x), t)=0
$$

has a unique solution $\bar{t}=\bar{s}(x)$. The mapping $\bar{s}: \Gamma \longrightarrow \mathbf{R}$ is Lipschitz and it is related with $\bar{u}$ through the formula

$$
\bar{u}(x)=\operatorname{Proj}_{[\alpha, \beta]}(\bar{s}(x))=\max \{\alpha, \min \{\beta, \bar{s}(x)\}\} .
$$

Moreover $\bar{u} \in C^{0,1}(\Gamma)$ and $\bar{y}, \bar{\varphi} \in W^{2, p}(\Omega) \subset C^{0,1}(\bar{\Omega})$ for some $p>2$.

In order to establish the second order optimality conditions we define the cone of critical directions. The derivative of $J$ can be represented by the function in $L^{2}(\Gamma)$ :

$$
\bar{d}(x)=\frac{\partial l}{\partial u}(x, \bar{y}(x), \bar{u}(x))+\bar{\varphi}(x) .
$$

The cone is:

$$
\begin{gathered}
C_{\bar{u}}=\left\{v \in L^{2}(\Gamma) \text { satisfying }(7) \text { and } v(x)=0 \text { if }|\bar{d}(x)|>0\right\}, \\
v(x)= \begin{cases}\geq 0 & \text { for a.e. } x \in \Gamma \text { where } \bar{u}(x)=\alpha, \\
\leq 0 & \text { for a.e. } x \in \Gamma \text { where } \bar{u}(x)=\beta\end{cases}
\end{gathered}
$$

Now we formulate the second order necessary and sufficient optimality conditions. See Casas and Mateos [4] 
THEOREM 5 If $\bar{u}$ is a local solution of $(P)$, then $J^{\prime \prime}(\bar{u}) v^{2} \geq 0$ holds for all $v \in C_{\vec{u}}$. Conversely, if $\bar{u} \in U^{\text {ad }}$ satisfies the first order optimality conditions (3)-(5) and the coercivity condition $J^{\prime \prime}(\bar{u}) v^{2}>0$ holds for all $v \in C_{\bar{u}} \backslash\{0\}$, then there exist $\delta>0$ and $\varepsilon>0$ such that

$$
J(u) \geq J(\bar{u})+\delta\|u-\bar{u}\|_{L^{2}(\Gamma)}^{2}
$$

is satisfied for every $u \in U^{a d}$ such that $\|u-\bar{u}\|_{L^{\infty}(\Omega)} \leq \varepsilon$.

\section{Discretization}

Here, we define a finite-element based approximation of the optimal control problem $(P)$. To this aim, we consider a regular family of triangulations $\left\{\mathcal{T}_{h}\right\}_{h>0}$ of $\bar{\Omega}: \bar{\Omega}=\cup_{T \in \mathcal{T}_{h}} T$.

For fixed $h>0$, we denote by $\left\{T_{j}\right\}_{j=1}^{N(h)}$ the family of triangles of $\mathcal{T}_{h}$ with a side on the boundary of $\Gamma$. If the edges of $T_{j} \cap \Gamma$ are $x_{\Gamma}^{j}$ and $x_{\Gamma}^{j+1}$ then $\left[x_{\Gamma}^{j}, x_{\Gamma}^{j+1}\right]:=T_{j} \cap \Gamma, 1 \leq j \leq N(h)$, with $x_{\Gamma}^{N(h)+1}=x_{\Gamma}^{1}$.

\subsection{Discretization of the state equation}

Associated with this triangulation we set

$$
Y_{h}=\left\{y_{h} \in C(\bar{\Omega}) \mid y_{h \mid T} \in \mathcal{P}_{1}, \text { for all } T \in \mathcal{T}_{h}\right\},
$$

where $\mathcal{P}_{1}$ is the space of polynomials of degree less than or equal to 1 . For each $u \in L^{\infty}(\Gamma)$, we denote by $y_{h}(u)$ the unique element of $Y_{h}$ that satisfies

$$
a\left(y_{h}(u), z_{h}\right)=\int_{\Omega} a_{0}\left(x, y_{h}(u)\right) z_{h} d x+\int_{\Gamma}\left[b_{0}\left(x, y_{h}(u)\right)+u\right] z_{h} d x \forall z_{h} \in Y_{h},
$$

where $a: Y_{h} \times Y_{h} \longrightarrow \mathbf{R}$ is the bilinear form defined by

$$
a\left(y_{h}, z_{h}\right)=\int_{\Omega} \nabla y_{h}(x) \nabla z_{h}(x) d x .
$$

The existence and uniqueness of a solution of (8) follows in the standard way from the monotonicity of $a_{0}$ and $b_{0}$ (see [7]).

Let us now introduce the approximate adjoint state associated to a control. To every $u \in U_{a d}$ we relate $\varphi_{h}(u) \in Y_{h}$, the unique function satisfying

$$
\begin{gathered}
a\left(\varphi_{h}(u), z_{h}\right)=\int_{\Omega}\left(\frac{\partial a_{0}}{\partial y}\left(x, y_{h}(u)\right) \varphi_{h}(u)+\frac{\partial L}{\partial y}\left(x, y_{h}(u)\right)\right) z_{h} d x+ \\
\int_{\Gamma}\left(\frac{\partial b_{0}}{\partial y}\left(x, y_{h}(u)\right) \varphi_{h}(u)+\frac{\partial l}{\partial y}\left(x, y_{h}(u), u\right)\right) z_{h} d \sigma(x) \quad \forall z_{h} \in Y_{h}
\end{gathered}
$$


The following approximation properties are essential to study the approximation of the control problem. They follow from real interpolation. See Brenner and Scott [2, Section 12.3] and [5]. Proof of inequality (9) is more technical. It is done adapting the proof of Aubin-Nietsche Lemma to semilinear equations as in [5] and taking into account the $H^{3 / 2}(\Omega)$ regularity of the solution of the Neumann problem with data in $L^{2}(\Gamma)$. A full proof will appear in the forthcoming paper [6].

Theorem 6 (i) For every $u \in H^{1 / 2}(\Gamma)$ there exists $C>0$, depending continuously on $\|u\|_{H^{1 / 2}(\Gamma)}$, such that

$$
\left\|y_{u}-y_{h}(u)\right\|_{H^{s}(\Omega)}+\left\|\varphi_{u}-\varphi_{h}(u)\right\|_{H^{s}(\Omega)} \leq C h^{2-s} \text { for all } 0 \leq s \leq 1,
$$

and

$$
\left\|y_{u}-y_{h}(u)\right\|_{L^{2}(\Gamma)}+\left\|\varphi_{u}-\varphi_{h}(u)\right\|_{L^{2}(\Gamma)} \leq C h^{3 / 2}
$$

(ii) For every $u \in L^{2}(\Gamma)$ there exists $C_{0}>0$, depending continuously on $\|u\|_{L^{2}(\Gamma)}$, such that

$$
\left\|y_{u}-y_{h}(u)\right\|_{H^{s}(\Omega)}+\left\|\varphi_{u}-\varphi_{h}(u)\right\|_{H^{s}(\Omega)} \leq C_{0} h^{3 / 2-s} \text { for all } 0 \leq s \leq 1 .
$$

(iii) For every $u_{1}, u_{2} \in L^{2}(\Gamma)$ there exists a constant $C>0$ such that

$$
\begin{gathered}
\left\|y_{u_{1}}-y_{u_{2}}\right\|_{H^{1}(\Omega)}+\left\|y_{h}\left(u_{1}\right)-y_{h}\left(u_{2}\right)\right\|_{H^{1}(\Omega)}+ \\
\left\|\varphi_{u_{1}}-\varphi_{u_{2}}\right\|_{H^{1}(\Omega)}+\left\|\varphi_{h}\left(u_{1}\right)-\varphi_{h}\left(u_{2}\right)\right\|_{H^{1}(\Omega)} \leq C\left\|u_{1}-u_{2}\right\|_{L^{2}(\Gamma)} .
\end{gathered}
$$

(iv) Moreover, if $u_{h} \rightarrow$ uweakly in $L^{2}(\Gamma)$, then $y_{h}\left(u_{h}\right) \rightarrow y_{u}$ and $\varphi_{h}\left(u_{h}\right) \rightarrow$ $\varphi_{u}$ strongly in $C(\bar{\Omega})$.

\subsection{Discrete optimal control problem}

We have several choices to write a discrete optimal control problem. Set

$$
\begin{gathered}
U_{h}^{0}=\left\{u \in L^{\infty}(\Gamma) \mid u_{\mid\left(x_{\Gamma}^{j}, x_{\Gamma}^{j+1}\right)} \in \mathcal{P}_{0} \text { for } 1 \leq j \leq N(h)\right\}, \\
U_{h}^{1}=\left\{u \in C(\Gamma) \mid u_{\mid\left(x_{\Gamma}^{j}, x_{\Gamma}^{j+1}\right)} \in \mathcal{P}_{1} \text { for } 1 \leq j \leq N(h)\right\} .
\end{gathered}
$$

and, following Hinze [9], we can semidiscretize the problem and take $U_{h}^{2}=$ $L^{2}(\Gamma)$. The corresponding approximated control problems are, for $i \in\{0,1,2\}$, $\left(\mathrm{P}_{h}^{i}\right)\left\{\begin{array}{l}\min J_{h}\left(u_{h}\right)=\int_{\Omega} L\left(x, y_{h}\left(u_{h}\right)(x)\right) d x+\int_{\Gamma} l\left(x, y_{h}\left(u_{h}\right)(x), u_{h}(x)\right) d \sigma(x), \\ \text { subject to }\left(y_{h}\left(u_{h}\right), u_{h}\right) \in Y_{h} \times U_{h}^{a d, i} \text { satysfying }(8),\end{array}\right.$ 
where $U_{h}^{a d, i}=U_{h}^{i} \cap U^{a d}$.

The first order optimality conditions can be written as follows:

THEOREM 7 Fix $i \in\{0,1,2\}$ and assume that $\bar{u}_{h}$ is a local optimal solution of $\left(P_{h}^{i}\right)$. Then there exist $\bar{y}_{h}$ and $\bar{\varphi}_{h}$ in $Y_{h}$ satisfying

$$
\begin{gathered}
a\left(\bar{y}_{h}, z_{h}\right)=\int_{\Omega} a_{0}\left(x, \bar{y}_{h}\right) z_{h} d x+\int_{\Gamma}\left(b_{0}\left(x, \bar{y}_{h}\right)+\bar{u}_{h}\right) z_{h} d x \quad \forall z_{h} \in Y_{h}, \\
a\left(\bar{\varphi}_{h}, z_{h}\right)=\int_{\Omega}\left(\frac{\partial a_{0}}{\partial y}\left(x, \bar{y}_{h}\right) \bar{\varphi}_{h}+\frac{\partial L}{\partial y}\left(x, \bar{y}_{h}\right)\right) z_{h} d x+ \\
\int_{\Gamma}\left(\frac{\partial b_{0}}{\partial y}\left(x, \bar{y}_{h}\right) \bar{\varphi}_{h}+\frac{\partial l}{\partial y}\left(x, \bar{y}_{h}, \bar{u}_{h}\right)\right) z_{h} d \sigma(x) \quad \forall z_{h} \in Y_{h}, \\
\int_{\Gamma}\left(\bar{\varphi}_{h}+\frac{\partial l}{\partial u}\left(x, \bar{y}_{h}, \bar{u}_{h}\right)\right)\left(u_{h}-\bar{u}_{h}\right) d \sigma(x) \geq 0 \quad \forall u_{h} \in U_{h}^{a d, i} .
\end{gathered}
$$

REMARK 8 At this point, we can show the difficulty introduced by the fact that $U_{h}^{1}$ is formed by continuous piecewise linear functions instead of piecewise constant functions. To make a clear presentation, let us assume for a while that $l(x, y, u)=\ell(x, y)+\frac{\Lambda}{2} u^{2}$. In the case where $U_{h}^{0}$ is formed by piecewise constant functions, we get from (10) that

$$
\bar{u}_{h !\left(x_{\Gamma}^{j}, x_{\Gamma}^{j+1}\right)}=\operatorname{Proj}_{[\alpha, \beta]}\left(-\frac{1}{\Lambda} \int_{x_{\Gamma}^{j}}^{x_{\Gamma}^{j+1}} \bar{\varphi}_{h}(x) d \sigma(x)\right) .
$$

Comparing this representation of $\bar{u}_{h}$ with (6) we can prove that $\bar{u}_{h} \rightarrow \bar{u}$ strongly in $L^{\infty}(\Gamma)$; see [7].

Since we are considering piecewise linear controls in the present paper, no such pointwise projection formula can be deduced. We only can say that $\bar{u}_{h}$ is the convex projection of $-\frac{1}{\Lambda} \bar{\varphi}_{h}(x)$. More precisely, $\bar{u}_{h}$ is the solution of problem

$$
\min _{v_{h} \in U_{h}}\left\|\bar{\varphi}_{h}+\Lambda v_{h}\right\|_{L^{2}(\Gamma)}^{2}
$$

This makes the analysis of the convergence more difficult than in [7]. In particular, we can prove that $\bar{u}_{h} \rightarrow \bar{u}$ strongly in $L^{2}(\Gamma)$, but this convergence cannot be obtained in $L^{\infty}(\Gamma)$ in an easy way as done in [7]. The reader is also referred to [8] for the study of problem (11).

We next can state a convergence result.

THEOREM 9 Fix $i \in\{0,1,2\}$. For every $h>0$ let $\bar{u}_{h}$ be a solution of $\left(P_{h}^{i}\right)$. Then there exist subsequences $\left\{\bar{u}_{h}\right\}_{h>0}$ converging in the weak* topology of $L^{\infty}(\Gamma)$ that will be denoted in the same way. If $\bar{u}_{h} \rightarrow \bar{u}$ in the mentioned topology, then $\bar{u}$ is a solution of $(P)$ and

$$
\lim _{h \rightarrow 0} J_{h}\left(\bar{u}_{h}\right)=J(\bar{u}) \text { and } \lim _{h \rightarrow 0} \| \bar{u}-\left.\bar{u}_{h}\right|_{L^{2}(\Gamma)}=0 .
$$


Moreover, for $i \in\{0,2\}, \lim _{h \rightarrow 0}\left\|\bar{u}-\bar{u}_{h}\right\|_{L^{\infty}(\Gamma)}=0$.

The main result of the paper is the following.

THEOREM 10 Let $\bar{u}$ be a solution of problem $(P)$ such that $J^{\prime \prime}(\bar{u}) v^{2}>0$ holds for all $v \in C_{\bar{u}} \backslash\{0\}$ and $\bar{u}_{h}^{i}$ a sequence of solutions of $\left(P_{h}^{i}\right)$ converging in $L^{2}(\Gamma)$ to $\bar{u}$. Then

1 There exists a constant $C>0$ and $h_{0}>0$ such that for $0<h<h_{0}$ $\left\|\bar{u}-\bar{u}_{h}^{0}\right\|_{L^{2}(\Gamma)} \leq C h$;

$2 \lim _{h \rightarrow 0} \frac{\left\|\bar{u}-\bar{u}_{h}^{1}\right\|_{L^{2}(\Gamma)}}{h}=0$;

3 For every $0<\varepsilon<1 / 2$ there exists a constant $C>0$ and $h_{0}>0$ such that for $0<h<h_{0},\left\|\bar{u}-\bar{u}_{h}^{2}\right\|_{L^{2}(\Gamma)} \leq C h^{3 / 2-\varepsilon}$.

In many practical cases when we make the full discretization using continuous piecewise linear controls $(i=1)$, the order of convergence observed for the controls in $L^{2}(\Gamma)$ is $h^{3 / 2}$. Let us show why. We will make two assumptions that are fulfilled in many situations:

(Q1) $l(x, y, u)=\ell(x, y)+e(x) u+\frac{\Lambda}{2} u^{2}$, where $\Lambda>0$ and

- the function $\ell: \Gamma \times \mathbf{R} \longrightarrow \mathbf{R}$ is Lipschitz with respect to the first component, of class $C^{2}$ with respect to the second variable, $\ell(\cdot, 0) \in$ $L^{1}(\Gamma), \frac{\partial^{2} \ell}{\partial y^{2}}(\cdot, 0) \in L^{\infty}(\Gamma)$ and for all $M>0$ there exists a constant $C_{\ell, M}>0$ such that

$$
\begin{gathered}
\left|\frac{\partial \ell}{\partial y}\left(x_{2}, y\right)-\frac{\partial \ell}{\partial y}\left(x_{1}, y\right)\right| \leq C_{\ell, M}\left|x_{2}-x_{1}\right|, \\
\left|\frac{\partial^{2} \ell}{\partial y^{2}}\left(x, y_{2}\right)-\frac{\partial^{2} \ell}{\partial y^{2}}\left(x, y_{1}\right)\right| \leq C_{\ell, M}\left|y_{2}-y_{1}\right|,
\end{gathered}
$$

for a.e. $x, x_{i} \in \Gamma$ and $|y|,\left|y_{i}\right| \leq M, i=1,2$;

- the function $e: \Gamma \rightarrow \mathbf{R}$ is Lipschitz and satisfies the following approximation property: there exists $C_{e}>0$ such that $\left\|e-\Pi_{h} e\right\|_{L^{2}(\Gamma)} \leq C_{e} h^{3 / 2}$. This assumption is not very constraining. Although it is not true for Lipschitz functions in general, it is true for a very wide class of functions. For instance for Lipschitz functions that are piecewise in $H^{3 / 2}(\Gamma)$.

(Q2) If we name $\Gamma_{s}=\{x \in \Gamma: \bar{u}(x)=\alpha$ or $\vec{u}(x)=\beta\}$, then the number of points in $\partial \Gamma_{s}$-the boundary of $\Gamma_{s}$ in the topology of $\Gamma-$ is finite. 
THEOREM 11 Suppose $(Q 1)$ and $(Q 2)$ are satisfied. Let $\vec{u}$ be a solution of problem $(P)$ such that $J^{\prime \prime}(\bar{u}) v^{2}>0$ holds for all $v \in C_{\bar{u}} \backslash\{0\}$ and $\bar{u}_{h} a$ sequence of solutions of $\left(P_{h}^{1}\right)$ converging in $L^{2}(\Gamma)$ to $\bar{u}$. Then there exists $C>0$ such that

$$
\left\|\bar{u}_{h}-\bar{u}\right\|_{L^{2}(\Gamma)} \leq C h^{3 / 2}
$$

REMARK 12 Using the inverse inequality

$$
\left\|u_{h}\right\|_{L^{\infty}(\Gamma)} \leq C h^{-1 / 2}|| u_{h} \|_{L^{2}(\Gamma)} \text { for all } u_{h} \in U_{h}^{1}
$$

We can get error estimates for continuous piecewise linear approximations for the control. This is important, since we have not been able to establish even uniform convergence up to now

About the proof of Theorem 10. For $i=0$ see [7]. Using second order sufficient conditions, we prove that there exists $\nu>0$ and $h_{1}>0$ such that for all $0<h<h_{1}$

$$
\nu\left\|\bar{u}-\bar{u}_{h}\right\|_{L^{2}(\Gamma)}^{2} \leq\left(J^{\prime}\left(\bar{u}_{h}\right)-J^{\prime}(\bar{u})\right)\left(\bar{u}_{h}-\bar{u}\right) .
$$

This is the most difficult part since we do not have uniform convergence of the states and we cannot apply the same techniques as in $[3,7]$.

Using (12) and first order optimality conditions (5) and (10) we have that

$$
\begin{aligned}
& \nu\left\|\bar{u}_{h}-\bar{u}\right\|_{L^{2}(\Gamma)}^{2} \leq\left(J_{h}^{\prime}\left(\bar{u}_{h}\right)-J^{\prime}(\bar{u})\right)\left(u_{h}^{*}-\bar{u}\right)+ \\
& +J^{\prime}(\bar{u})\left(u_{h}^{*}-\bar{u}\right)+\left(J_{h}^{\prime}\left(\bar{u}_{h}\right)-J^{\prime}\left(\bar{u}_{h}\right)\right)\left(\bar{u}-\bar{u}_{h}\right) .
\end{aligned}
$$

For $i=1, u_{h}^{*}=\Pi_{h} \bar{u}$ is the unique function in $U_{h}^{1}$ such that $\Pi_{h} \bar{u}\left(x_{\Gamma}^{j}\right)=$ $\bar{u}\left(x_{\Gamma}^{j}\right)$ for $j=1, \ldots, N(h)$. In this case first and second terms are of order $o\left(h^{2}\right)$. For $i=2$ (semidiscretization), $u_{h}^{*}=\bar{u}$ and the first two terms are zero. Third term is more difficult. Since we do not know yet if $\left\{\bar{u}_{h}\right\}$ is bounded in $H^{1 / 2}(\Gamma)$ a direct proof as in the distributed case (see [3]) would lead to a bad estimate. With a small turnaround, we can prove that for every $\rho>0$ and every $0<\varepsilon \leq 1 / 2$ there exists $C_{\rho, \varepsilon}>0$ independent of $h$ such that the third term can be estimated by

$$
\left(C_{\rho, \varepsilon} h^{3 / 2-\varepsilon}+\rho\left\|\bar{u}_{h}-\bar{u}\right\|_{L^{2}(\Gamma)}\right)\left\|\bar{u}_{h}-\bar{u}\right\|_{L^{2}(\Gamma)} .
$$

We must take $\rho$ small enough to conclude the proof. $\square$

\section{References}

[1] N. Arada, E. Casas, and F. Tröltzsch. Error estimates for the numerical approximation of a semilinear elliptic control problem. Comput. Optim. Appl., 23(2):201-229, 2002. 
[2] S. C. Brenner and L. R. Scott. The mathematical theory of finite element methods, volume 15 of Texts in Applied Mathematics. Springer-Verlag, New York, 1994.

[3] E. Casas. Using piecewise linear functions in the numerical approximation of semilinear elliptic control problems. AiCM, 2005.

[4] E. Casas and M. Mateos. Second order optimality conditions for semilinear elliptic control problems with finitely many state constraints. SIAM J. Control Optim., 40(5):1431-1454 (electronic), 2002.

[5] E. Casas and M. Mateos. Uniform convergence of the FEM. Applications to state constrained control problems. Comput. Appl. Math., 21(1):67-100, 2002. Special issue in memory of Jacques-Louis Lions.

[6] E. Casas and M. Mateos. Error estimates for the numerical approximation of Neumann control problems. Submitted.

[7] E. Casas, M. Mateos, and F. Tröltzsch. Error estimates for the numerical approximation of boundary semilinear elliptic control problems. Computational Optimization and Applications, 31(2):193-219, 2005.

[8] E. Casas and J.-P. Raymond. The stability in $W^{s, p}(\Gamma)$ spaces of the $L^{2}$-projections on some convex sets of finite element function spaces. To appear.

[9] M. Hinze. A variational discretization concept in control constrained optimization: the linear-quadratic case. Comput. Optim. Appl., 30(1):45-61, 2005.

[10] C. Meyer and A. Rösch. Superconvergence properties of optimal control problems. SIAM Journal on Control and Optimization, 43(3):970-985, 2005. 